\title{
ESTUDO DA SEPARAÇÃO DE TERRAS RARAS USANDO SISTEMAS EXTRATANTES ORGANOFOSFORADOS
}

\author{
M. NASCIMENTO ${ }^{1}$, Y.M. VERA ${ }^{1}$, B. M. VALVERDE ${ }^{2}$ e R. C. GOMES ${ }^{2}$ \\ ${ }^{1}$ Centro de Tecnologia Mineral, Coordenação de Processos Metalúrgicos e Ambientais \\ ${ }^{2}$ Universidade Federal do Rio de Janeiro , Escola de Química \\ E-mail para contato: marisa@cetem.gov.br
}

\begin{abstract}
RESUMO - O presente trabalho apresenta os resultados ou a análise das variáveis de processo para a separação de terras raras leves e pesadas, em meio clorídrico, por extração por solventes. Reagentes organofosforados e isoparafina foram usados como sistemas de extração e o licor foi produzido em laboratório por dissolução de óxidos de terras raras em HCl para simular um licor de lixiviação proveniente de minérios monazíticos, após a remoção do cério. Os seguintes parâmetros foram determinados: $\mathrm{pH}$ de trabalho e concentração do extratante. Um circuito contínuo de extração foi operado e seu comportamento foi similar aos apresentados nos testes em batelada.
\end{abstract}

\section{INTRODUÇÃO}

Os 17 elementos conhecidos com terras raras (ETR) consistem em um grupo de 15 elementos, chamados de lantanídeos, o ítrio e o escândio que, apesar de não constarem no grupo dos lantanídeos apresentam propriedades similares e ocorrências geológicas comuns.Todos esses elementos ocorrem juntos na tabela periódica, no entanto, podemos dividi-los em duas categorias: as terras raras leves: lantânio $(\mathrm{La})$, cério $(\mathrm{Ce})$, praseodímio $(\mathrm{Pr})$ e neodímio $(\mathrm{Nd})$; e, terras raras pesadas: samário $(\mathrm{Sm})$, európio $(\mathrm{Eu})$, gadolínio $(\mathrm{Gd})$, térbio $(\mathrm{Tb})$, disprósio (Dy), hólmio (Ho), érbio (Er), túlio $(\mathrm{Tm})$, itérbio $(\mathrm{Yb})$, lutécio $(\mathrm{Lu})$ e ítrio ( Y) (AIMAN GOLD COORPORATION, 2012).

A separação e recuperação dos ETR mais ítrio tem sido assunto de muitos pesquisadores por várias décadas. No passado as separações desses elementos eram realizadas através de sucessivas recristalizações e precipitações. Hoje a extração por solventes é a operação unitária mais utilizada, no entanto, ainda com a dificuldade de separação que se dá pelas propriedades químicas similares dessa série de elementos onde os fatores de separação(F) ${ }^{1}$ entre os ETR adjacentes são muito pequenos.

No Brasil, as principais fontes minerais de terras raras contém principalmente as terras raras leves (La, Ce, Pr e Nd) como é o caso das ocorrências em Catalão(GO), Araxá(MG) e litoral do Rio

\footnotetext{
${ }^{1}$ Fator de separação (F) - Para a situação entre dois metais onde a separação deve ser realizada a partir de uma mesma solução, o fator de separação é definido como a razão entre os coeficientes de extração de cada metal (D), que, por sua vez é dado pela razão entre a concentração do metal na fase orgânica e na fase aquosa.
} 


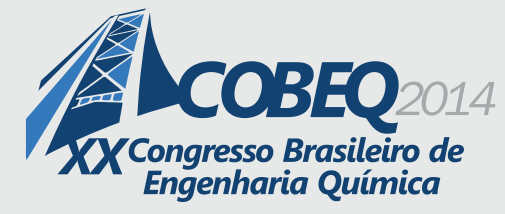

19 a 22 de outubro de 2014

Florianópolis/SC

de Janeiro. Ocorrências de ETR pesadas são relatadas na área da mina de Pitinga em Presidente Figueiredo (AM) (Lapido-Loureiro, 1994).

A operação de separação líquido-líquido ou extração por solventes (SX), aplicada à separação de ETR, consiste em essência no tratamento de uma fase aquosa contendo um ou mais íons em solução, com uma fase orgânica denominada solvente capaz de extrair seletivamente aquelas espécies de interesse (Ritcey \& Ashbrook, 1984). Formam-se assim os chamados "organocomplexos" dos metais extraídos que são contidos na fase orgânica, permitindo sua remoção do sistema (Marcus \& Kertes, 1969). A etapa seguinte (re-extração ou esgotamento) consiste na reversão desta reação de extração trazendo novamente o elemento de interesse da fase orgânica carregada para uma nova fase aquosa, desta vez adequada a um posterior tratamento de recuperação. A etapa de re-extração também pode ser conduzida de forma a obter seletividade entre os elementos removidos da fase orgânica (Ritcey \& Ashbrook, 1984). Com a combinação das etapas de extração e re-extração é alcançado o fracionamento e a purificação das soluções obtidas da operação.

A fase aquosa contendo os ETR tem características fortemente associadas às fontes minerais tratadas e ao processamento anterior adotado para a dissolução dos concentrados sólidos disponíveis. Assim, identificam-se os meios clorídrico, nítrico e sulfúrico como os mais freqüentemente adotados para o fracionamento por SX. A cada um destes meios associam-se características particulares das espécies iônicas de TRs presentes que tornam mais adequada a aplicação de um tipo de extratante orgânico (Soares, 1994, Thakur, 2000). Em grande parte da literatura o objetivo principal é a produção de ETR e seus fracionamentos utilizando solventes fosforados como o D2EHPA, Ionquest 801 e Cyanex 272.

Neste trabalho, dois extratantes foram avaliados e comparados, o D2EHPA (ácido di(2-etilhexil)fosfórico) e o P-507 (ácido di(2-etil-hexil)fosfônico) na separação das terras raras em frações leve e pesada contidas em uma solução clorídrica. Para tal, foram realizados ensaios de bancada e piloto.

\section{MATERIAS E MÉTODOS}

O licor sintético de cloretos de TR foi preparado, à quente, a partir de seus óxidos através da adição de $\mathrm{HCl}$ com excesso estequiométrico. Antes da adição do $\mathrm{HCl}$ deve-se fazer uma pasta da mistura de óxidos com adição de um pouco de água destilada em um bécher, para facilitar a solubilização do mesmo. Após o preparo dessa pasta coloca-se o bécher em uma chapa de aquecimento e adiciona-se o $\mathrm{HCl}$ aos poucos. O cloreto estará completamente formado quando a solução ficar límpida. Após esse processo o bécher deve ser mantido em aquecimento para redução do volume inicial de ácido e água até formação de uma pasta úmida e compacta. A pasta final foi então transferida para um balão volumétrico que, em seguida, foi avolumado com água destilada. A tabela 1 mostra as quantidades de cada óxido de ETR e ítrio utilizadas para a preparação do licor sintético baseado num antigo relatório da empresa Nuclemon. A ausência de Cério no licor foi planejada com o objetivo de simular um licor que sofreu um tratamento anterior de precipitação para a remoção do Cério contido como tratado na literatura (Awwal \& Figueiras, 1988). 
Tabela 1 - Composição do licor sintético isento de Cério tendo [TRtotais] 50g/L (Awwal \& Figueiras, 1988).

\begin{tabular}{|c|c|c|c|c|c|}
\hline \multirow[t]{2}{*}{ Componentes } & \multirow[t]{2}{*}{ PM } & \multirow{2}{*}{$\begin{array}{c}{\left[\mathrm{TR}_{2} \mathrm{O}_{3}\right]} \\
\mathrm{Mol} / \mathrm{L}\end{array}$} & \multirow{2}{*}{$\begin{array}{l}{\left[\mathrm{TR}^{3+}\right]} \\
\mathrm{Mol} / \mathrm{L}\end{array}$} & \multicolumn{2}{|c|}{ Alimentação } \\
\hline & & & & $\begin{array}{l}\mathrm{g} / \mathrm{L} \\
\mathrm{TR}_{2} \mathrm{O}_{3}\end{array}$ & $\begin{array}{l}\% \\
\mathrm{TR}_{2} \mathrm{O}_{3}\end{array}$ \\
\hline $\mathrm{La}_{2} \mathrm{O}_{3}$ & 325,82 & $6,45 \mathrm{E}-02$ & $1,29 \mathrm{E}-01$ & 21,02 & 42,04 \\
\hline $\mathrm{Pr}_{6} \mathrm{O}_{11}$ & 1021,4 & 4,77E-03 & $2,86 \mathrm{E}-02$ & 4,87 & 9,74 \\
\hline $\mathrm{Nd}_{2} \mathrm{O}_{3}$ & 336,48 & 5,19E-02 & $1,04 \mathrm{E}-01$ & 17,47 & 34,94 \\
\hline $\mathrm{Sm}_{2} \mathrm{O}_{3}$ & 348,7 & 6,93E-03 & 1,39E-02 & 2,42 & 4,84 \\
\hline $\mathrm{Eu}_{2} \mathrm{O}_{3}$ & 352,0 & $9,04 \mathrm{E}-04$ & 1,81E-03 & 0,32 & 0,64 \\
\hline $\mathrm{Gd}_{2} \mathrm{O}_{3}$ & 362,6 & $4,19 \mathrm{E}-03$ & 8,37E-03 & 1,52 & 3,04 \\
\hline $\mathrm{Tb}_{4} \mathrm{O}_{7}$ & 747,6 & $4,92 \mathrm{E}-04$ & 1,97E-03 & 0,37 & 0,74 \\
\hline $\mathrm{Dy}_{2} \mathrm{O}_{3}$ & 373,0 & 1,26E-03 & 2,51E-03 & 0,47 & 0,94 \\
\hline $\mathrm{Ho}_{2} \mathrm{O}_{3}$ & 377,8 & $5,78 \mathrm{E}-04$ & $1,16 \mathrm{E}-03$ & 0,22 & 0,44 \\
\hline $\mathrm{Er}_{2} \mathrm{O}_{3}$ & 382,6 & 5,70E-04 & $1,14 \mathrm{E}-03$ & 0,22 & 0,44 \\
\hline $\mathrm{Y}_{2} \mathrm{O}_{3}$ & 225,81 & 4,95E-03 & $9,90 \mathrm{E}-03$ & 1,12 & 2,24 \\
\hline \multicolumn{2}{|c|}{ SOMATÓRIO } & 1,41E-01 & 3,02E-01 & 50,0 & 100,0 \\
\hline \multicolumn{6}{|c|}{ Somatório de $\mathrm{TR}_{2} \mathrm{O}_{3}$ de médios e pesado (Sm ao Y) 1,99E-02 } \\
\hline \multicolumn{6}{|c|}{ Somatório de $\mathrm{TR}^{3+}$ de médios e pesado (Sm ao Y) 4,07E-02 } \\
\hline
\end{tabular}

Os sistemas extratantes trataram-se de soluções de D2EHPA (ácido di(2-etil-hexil)fosfórico) e P-507(ácido di(2-etil-hexil)fosfônico), fornecidos respectivamente pela RHODIA e Luoyang Aoda Chemical Co. Ltd, em diferentes concentrações. Para tal, procedeu-se a diluição dos extratantes em isoparafina 17/21, fornecida pela Ypiranga, nas concentrações indicadas em cada teste realizado. Foram realizados testes para verificação da influência do $\mathrm{pH}$ da solução inicial de terras raras e da concentração dos extratantes testados. Os ensaios de bancada foram realizados em bécheres de vidro com agitador magnético em placa de agitação da marca IKA. O pH foi controlado com um pHmetro da marca METRON. O tempo de agitação para todos os ensaios foi de 15 minutos. As fases descansaram durante 40 minutos para total separação em funis de decantação tipo pêra e depois levadas para a quantificação analítica dos ETR. As análises químicas das soluções aquosas foram realizadas por ICP-MS (ORIBA). As soluções orgânicas foram quantificadas por diferença.

O circuito piloto para a extração de ETR médios e pesados consistiu de um sistema de 6 misturadores-decantadores com volumes respectivos de $190 \mathrm{~mL}$ e $340 \mathrm{~mL}$ que operou em dias seguidos, em diferentes razões A/O e com a seguintes configurações: $\mathrm{pH}$ da alimentação de 1,0, 6 estágios; concentração do licor de 50 g/L de ETR totais e concentração dos extratantes em 20\% v/ $\mathrm{v}$ (volume por volume) em isoparafina.

\section{RESULTADOS}

A figura 1a mostra o comportamento do D2EHPA na extração dos elementos de TR leves mais o samário em função do $\mathrm{pH}$ inicial de trabalho. A análise do gráfico permitiu concluir que em pH em torno de 1,0 uma considerável separação entre o Sm e os ETR leves foi obtida. Os valores dos fatores de separação para o par de elementos $\mathrm{Sm} / \mathrm{Nd}$ e demais TRs podem ser consultados na tabela 2 . Os 


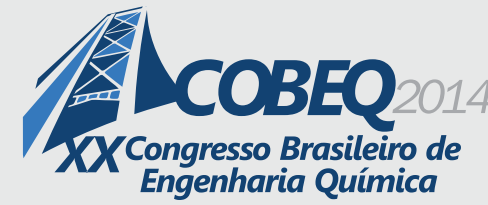

valores dos fatores de separação calculados através do teste de investigação do $\mathrm{pH}$ confirmam o valor de $\mathrm{pH}$ ideal de trabalho como sendo 1,04 , onde foi obtido o maior valor de $\mathrm{F}(7,86)$ para o par $\mathrm{Sm} / \mathrm{Nd}$, o que indica uma melhor separação dos ETR leves (La, Nd e Pr) em relação aos demais elementos.

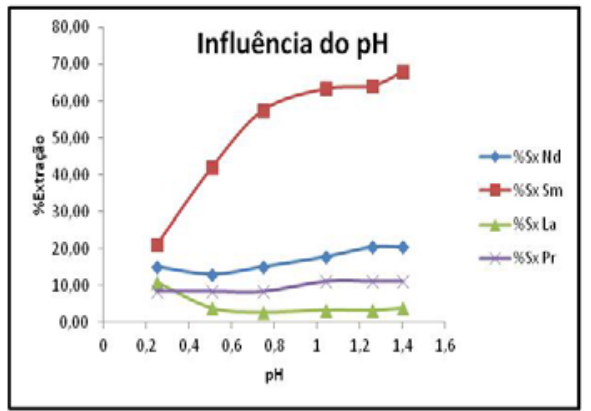

(a)

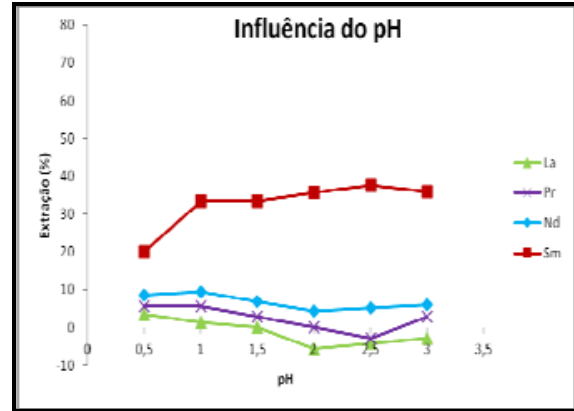

(b)

Figura 1 - Influência do pH na extração dos TRs para os extratantes D2EHPA (a) e P-507(b)

A Figura 1b mostra o comportamento de extração dos elementos de TRs leves mais o samário em função do $\mathrm{pH}$ inicial de trabalho para P-507. Inicialmente observam-se valores de extração muito menores dos que encontrados com o uso do D2EHPA. Observa-se também que para valores de $\mathrm{pH}$ iniciais da solução aquosa acima de 1,5 os elementos mais pesados estudados como o samário, são extraídos mais facilmente do que os outros elementos mais leves, melhorando assim bastante os valores dos fatores de separação F (DSm/SNd)(Tabela 2).

Tabela 2 - Fatores de separação dos pares de ETR em função do pH na extração com D2EHPA e P507

\begin{tabular}{|c|c|c|c|c|c|c|c|}
\hline \multicolumn{5}{|c|}{ D2EHPA } & \multicolumn{4}{c|}{ P-507 } \\
\hline $\begin{array}{c}\mathrm{pH} \\
\text { incial }\end{array}$ & $\begin{array}{c}\mathrm{F} \\
(\mathrm{DSm} / \mathrm{DNd})\end{array}$ & $\begin{array}{c}\mathrm{F} \\
(\mathrm{DNd} / \mathrm{DPr})\end{array}$ & $\begin{array}{c}\mathrm{F} \\
(\mathrm{DPr} / \mathrm{DLa})\end{array}$ & $\mathrm{pH}$ inicial & $\begin{array}{c}\mathrm{F} \\
(\mathrm{DSm} / \mathrm{DNd})\end{array}$ & $\begin{array}{c}\mathrm{F} \\
(\mathrm{DNd} / \mathrm{DPr})\end{array}$ & $\begin{array}{c}\mathrm{F} \\
(\mathrm{DPr} / \mathrm{DLa})\end{array}$ \\
\hline 1,40 & 8,12 & 2,08 & 3,13 & 0,5 & 2,68 & 1,59 & 1,64 \\
\hline 1,26 & 6,88 & 2,08 & 3,67 & 1,0 & 4,82 & 1,76 & 4,18 \\
\hline 1,04 & 7,86 & 1,76 & 3,67 & 1,5 & 6,81 & 2,57 & N.D \\
\hline 0,75 & 7,56 & 1,98 & 3,25 & 2,0 & 12,42 & N.D & 0,00 \\
\hline 0,51 & 4,87 & 1,65 & 2,28 & 2,5 & 11,15 & $-2,00$ & 0,68 \\
\hline 0,25 & 1,50 & 1,98 & 0,74 & 3,0 & 8,84 & 2,23 & $-1,06$ \\
\hline
\end{tabular}

Também é observado no uso do P-507 que para toda a faixa de $\mathrm{pH}$ estudada as extrações dos elementos mais leves (La, Ce, Pr e Nd) são menores que $10 \%$. 


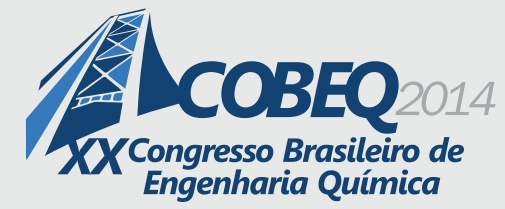

Em ambos extratantes usados, os baixos valores de fator de separação obtidos para os outros dois pares analisados ( $\mathrm{Pr} / \mathrm{La}$ e $\mathrm{Nd} / \mathrm{Pr}$ ) indicam a dificuldade de separação entre esse elementos em especial para a separação $\mathrm{Nd} / \mathrm{Pr}$.

Pode ser observado que em alguns casos não foi possivel a determinação dos fatores de separação (N.D) ou o valor foi igual a zero ou ainda negativo, porque, aparentemente, não houve extração para a fase orgânica ou, provavelmente causado por erro analítico.

A segunda etapa de ensaios de bancada teve como objetivo verificar qual a melhor concentração de trabalho dos extratantes testados através dos percentuais de extração dos ETR leves e do Sm. A partir dos dados de extração foi possível representar graficamente o percentual de extração em função da concentração dos extratantes como visto nas figuras $2 \mathrm{a}$ e $2 \mathrm{~b}$. Na figura $2 \mathrm{a}$, os melhores fatores de separação foram obtidos a partir das concentrações entre 20 e 25\% para D2EHPA.

Da análise do gráfico da figura $2 \mathrm{~b}$ pode ser concluído que, em $\mathrm{pH}$ inicial de trabalho igual a $1 \mathrm{e}$, para os elementos mais pesados (Sm), um aumento da concentração de P-507 produz um aumento dos percentuais de extração para a fase orgânica. No caso dos demais elementos investigados esse efeito é discreto. Os valores dos fatores de separação (F) do par Sm/Nd permitiram concluir que o melhor desempenho de separação foi obtido para as concentrações de P 507entre 20 e 25\%.

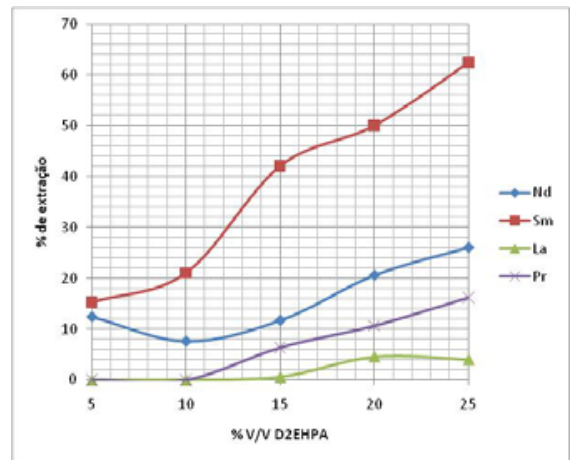

(a)

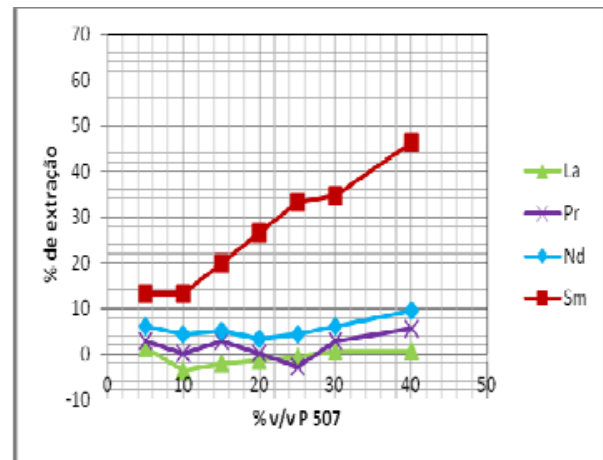

(b)

Figura 2 - Percentuais de extração de TRs em função da concentração de D2EHPA (a) e P-507 (b).

As figuras $3 a$ à $3 h$ apresentam os resultados de extração em circuito contínuo, para D2EHPA, por estágio, para os elementos $\mathrm{La}, \mathrm{Pr}, \mathrm{Nd}, \mathrm{Sm}$ e Gd. A entrada de alimentação aquosa ocorreu na célula número 6. Destacam-se as boas extrações de Sm e Gd já no primeiro estágio, enquanto que os demais metais, os leves, apresentam extração crescente ao longo dos estágios subseqüentes. A partir dos resultados analíticos foi possível determinar que o fator de separação entre ETRs médias e leves vai diminuindo ao longo dos estágios.

Nas figuras $4 a$ à $4 g$ apresentam os resultados de extração para os testes contínuos para P-507. Verifica-se inicialmente que para todas as relações de fase $\mathrm{A} / \mathrm{O}$ testadas, é apresentado a preferência de corte entre os elementos do par Gd-Sm. 


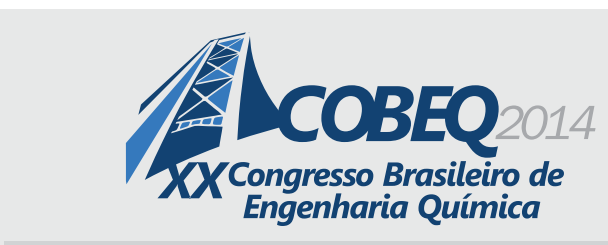

19 a 22 de outubro de 2014

Florianópolis/SC

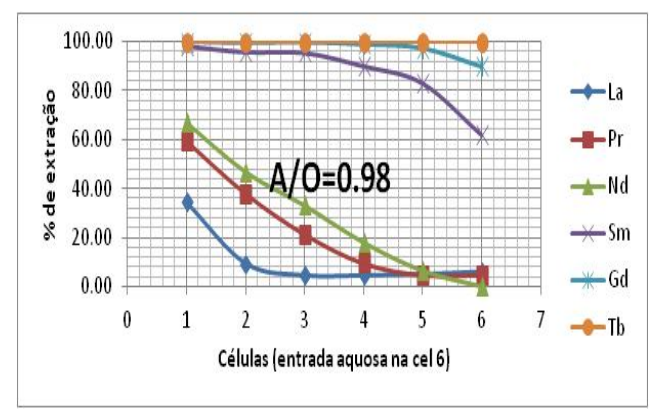

(a)

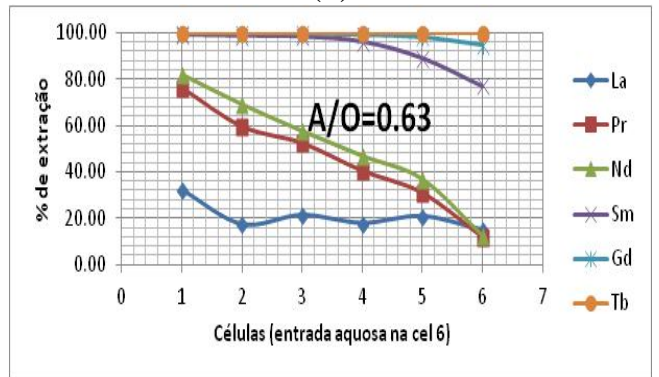

(c)

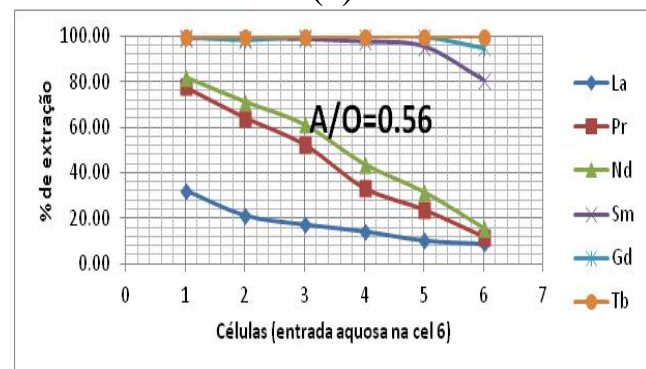

(e)

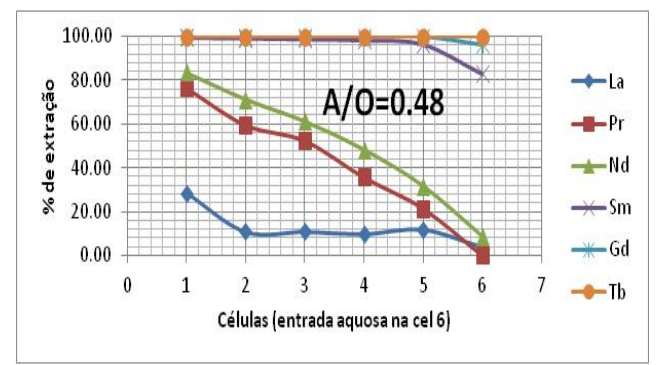

(g)

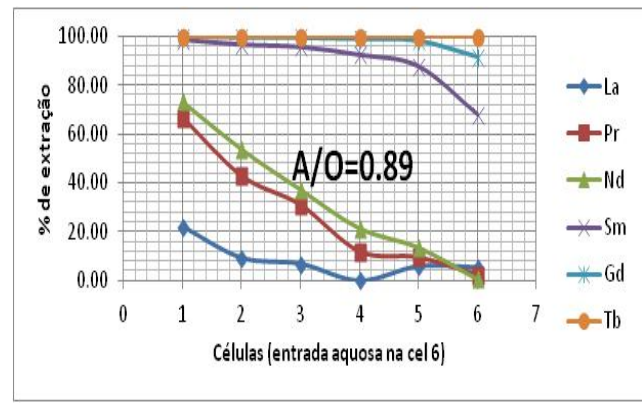

(b)

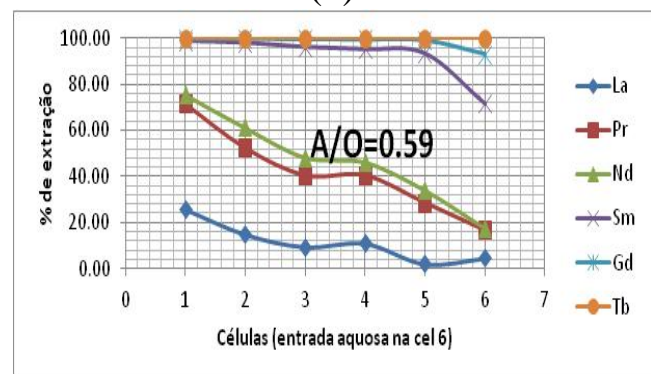

(d)

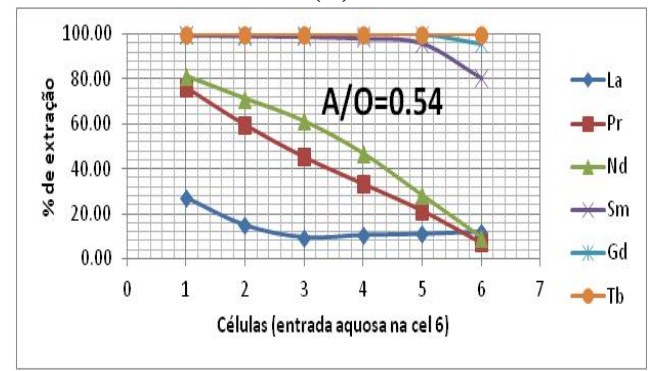

(f)

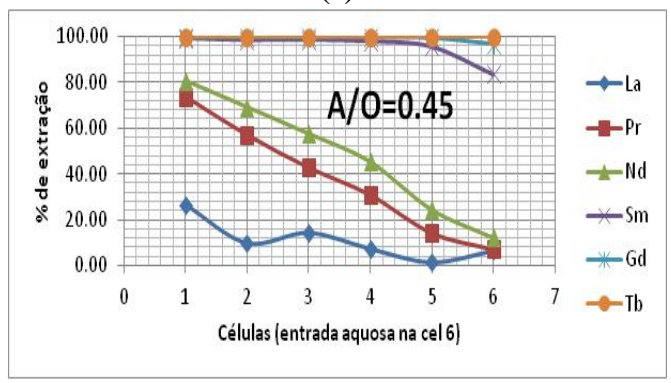

(h)

Figura 3- Resultados de extração, em diversos valores de razão aquoso-orgânico(a à h), para os testes contínuos em planta piloto utilizando D2EHPA como sistema extratante. pesados.

É importante notar que um aumento da razão A/O desfavorece a extração elementos mais 


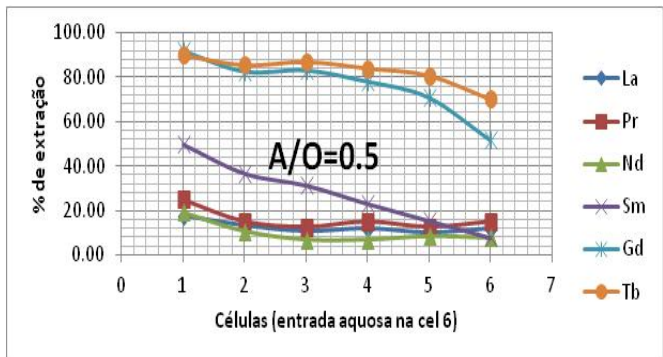

(a)

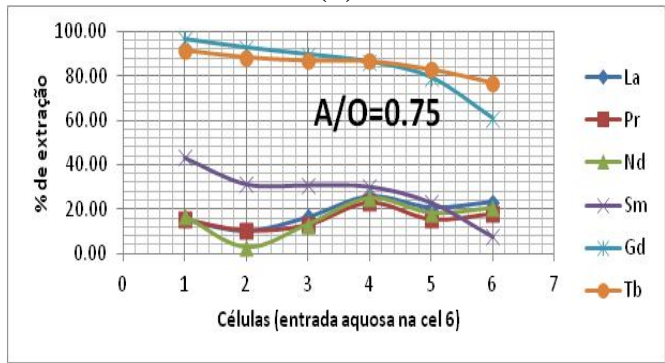

(c)

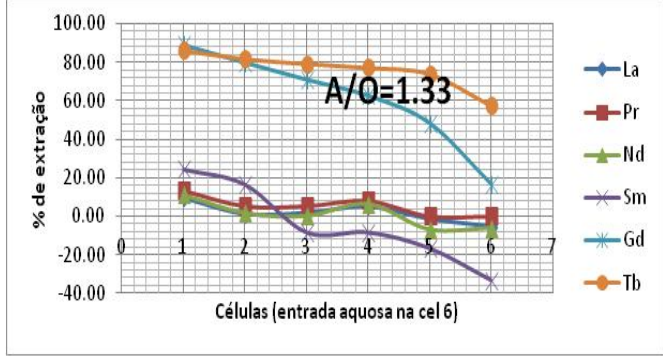

(e)

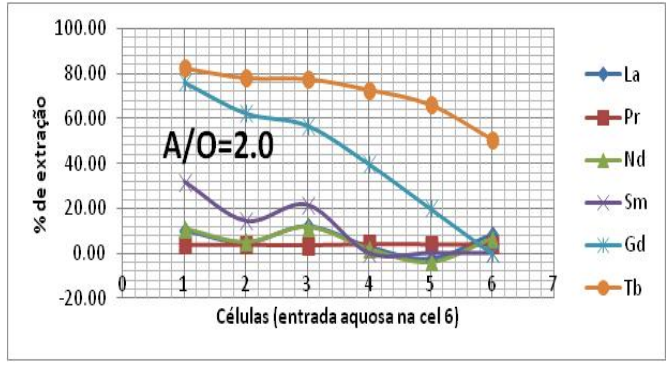

(g)

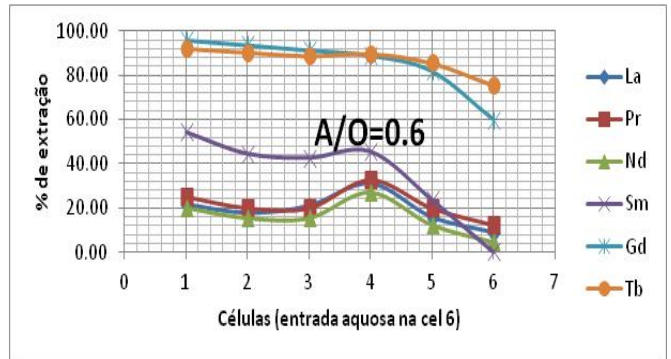

(b)

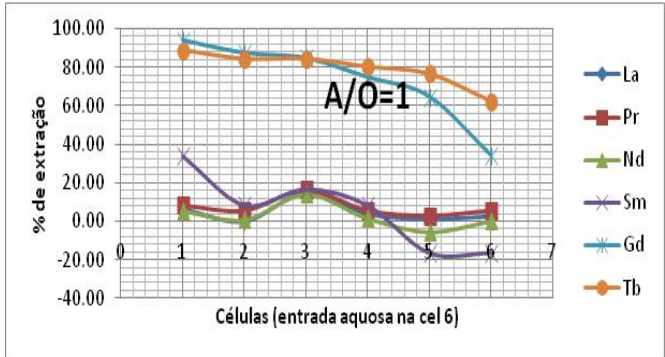

(d)

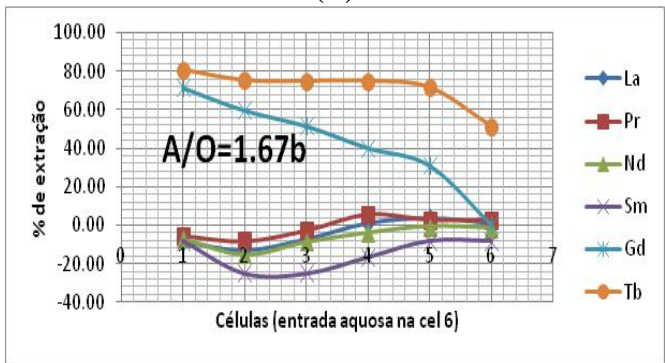

(f)

Figura 4 - Resultados de extração, em diversos valores de razão aquoso-orgânico(a à g), para os testes contínuos em planta piloto utilizando P-507 como sistema extratante. 


\section{CONCLUSÕES}

É possível extrair e separar ETRs em meio clorídrico utilizando os extratantes organofosforados D2EHPA e P-507. Comparando com o desempenho observado para ambos os extratantes foi possível concluir que o P-507 necessita de um valor de $\mathrm{pH}$ inicial mais elevado para apresentar um mesmo desempenho de extração pra Sm. No entanto os valores dos fatores de separação Sm-Nd parecem ser mais promissores para o P-507.

Nos dois casos, os valores de concentração do sistema de extração não apresentaram diferenças signifcativas sendo mantidas entre 20 e 25 \% V/V para melhor desempenho de separação Sm-Nd.

Os testes contínuos se mostraram condizentes com os resultados de bancada.

\section{AGRADECIMENTOS}

Os autores agradecem à RHODIA e ao CNPQ.

\section{REFERÊNCIAS}

- AIMAN GOLD COORPORATION. Rare Earth Elements 101. Rare Earth Elements (REE) Explained. April 2012. Page 3.

- Awwal, M. A., Filgueiras, S. A. C., SEPARAÇÃo E PURIFICAÇÃo dE GADOLínIO E OUTRAS TERRAS RARAS E ÍTRIO, Relatório de Progresso DETQ-010/88, Centro de Desenvolvimento da Tecnologia Nuclear, NUCLEBRAS - 1988.

- Lapido-Loureiro, F.E.V., 1994. Terras Raras no Brasil: depósitos, recursos identificados, reservas. CETEM, Rio de Janeiro, 102pp.

- Marcus, Y; Kertes, A.S. Ion Exchange and Solvent Extraction of Metal Complexes. London, John Wiley \& Sons, 1969 .

- Ritcey, G. M. ; Ashbrook, A.W. Solvent Extraction Principles and Applications to Process Metallurgy. Part I. N. York, Elsevier Science Publishers, 1984.

- Soares, P.S.M., (1994). Estudo preliminary do fracionamento de terras raras médias e pesadas em grupos com extração por solventes. Tese de mestrado, IPEN/USP.

- Thakur, N.V., (2000). Separation of Rare Earth by Solvent Extraction, In: Doyle, F.M., Arbiter, N., Gupta, C.K., (eds). Mineral Precessing and Extractive Metallurgy. Special Issue: Solvent Extraction in Production and processing of Nonferrous Metals - Part II. Volume 21. Number 1-5.pp. 277-307. 